\title{
RECUPERAÇÃO DE BROMO EM SOLUÇÕES AQUOSAS RESIDUAIS
}

\author{
Glauco Arnold Tavares*, José Albertino Bendassolli, Gleison de Souza, Felipe Rufine Nolasco, José Aurélio Bonassi e \\ Hugo Henrique Batagello \\ Centro de Energia Nuclear na Agricultura, Universidade de São Paulo, CP 96, 13400-970 Piracicaba - SP
}

Recebido em 9/4/03; aceito em 29/7/03

\begin{abstract}
BROMINE RECOVERY FROM WASTE AQUEOUS SOLUTIONS. A laboratory procedure was devised to recover bromine from waste alkaline aqueous solutions used in the isotopic determination of N-15. The laboratory apparatus comprises two round bottom flasks (1 and 2 L), a dropping funnel, a gas bubbler, a gas regulator and glass fittings. The waste solution is acidified with sulfuric acid forming molecular bromine that is stripped out by a flow of nitrogen gas bubbled through the solution. This gas is then bubbled through a solution of lithium hydroxide generating lithium bromide and lithium hypobromite. The efficiency of bromine recovery was estimated to be $82 \pm 2 \%$. This resulting solution was successfully reused in the isotopic determination of $\mathrm{N}-15$. The procedure can recycle most of the bromine used in the laboratory saving resources and preserving the environment. The procedure can be adapted to recover bromine of other laboratory waste streams.
\end{abstract}

Keywords: bromine recovery; residual solutions; residue treatment.

\section{INTRODUÇÃO}

A questão do gerenciamento de resíduos químicos, frutos de atividades de ensino e pesquisa, é um tema de discussão que vem cada vez mais ocupando espaço no meio acadêmico. Isso se justifica devido ao importante papel que universidades, escolas e institutos de pesquisas exercem na formação de recursos humanos acostumados às práticas de gestão ambiental, e também para servir de exemplo à sociedade na qual estão inseridos ${ }^{1-5}$.

Na implantação do Programa de Gerenciamento de Resíduos Químicos do Centro de Energia Nuclear na Agricultura (PGRQCENA/USP), identificou-se soluções de bromo, nas espécies brometo e hipobromito, em meio alcalino $(\mathrm{LiOH})$. Esse resíduo é de destacada importância do ponto de vista de toxidez, volume e valor agregado $\left(\mathrm{Br}_{2}\right)$ e, portanto, de interesse no desenvolvimento de processo de reciclagem. A referida solução é empregada e posteriormente gerada como resíduo na etapa de oxidação de espécies nitrogenadas a $\mathrm{N}_{2}$, no procedimento de determinação de ${ }^{15} \mathrm{~N}$ (\% átomos $)^{6}$. Ressalta-se que essa metodologia ainda é empregada em rotina no Laboratório de Isótopos Estáveis (LIE-CENA/USP), embora a grande maioria das análises através da técnica de espectrometria de massas venha sendo realizada empregando sistemas automatizados de preparo de amostras, onde esse resíduo não é mais gerado. Em contrapartida, os sistemas automatizados geram resíduos sólidos de óxido de cobre, cuja recuperação já vem sendo realizada no LIE-CENA/USP ${ }^{7}$.

Quando inalado ou ingerido, o bromo é extremamente tóxico, podendo causar edema pulmonar, entre outros efeitos ao organis$\mathrm{mo}^{8-10}$. Por outro lado, no estado líquido, possui significativo valor agregado, razões essas que levaram ao desenvolvimento de um sistema especial em vidro para uso na recuperação do bromo presente em soluções residuais. O procedimento adotado objetivou, após a acidificação da solução residual e conseqüente liberação do bromo na forma $\mathrm{Br}_{2}$, produzir novamente solução contendo $100 \mathrm{~g} \mathrm{~L}^{-1}$ de $\mathrm{Br}_{2}$ em LiOH $10 \%$ m/v, ou seja, a solução empregada em rotina na determinação de ${ }^{15} \mathrm{~N}$ (\% átomos) $)^{6}$.

*e-mail: gtavares@cena.usp.br
O estabelecimento deste procedimento ensejou, portanto, minimizar tanto o volume de resíduo químico produzido quanto seus possíveis efeitos nocivos ao meio ambiente, resultando ainda em retorno financeiro para a instituição.

\section{MATERIAL E MÉTODOS}

A solução empregada na determinação isotópica de ${ }^{15} \mathrm{~N}$ é obtida a partir da reação apresentada pela Equação (1)

$\mathrm{Br}_{2}(\mathrm{l})+2 \mathrm{LiOH}(\mathrm{aq}) \rightarrow \mathrm{LiBr}(\mathrm{aq})+\mathrm{LiBrO}(\mathrm{aq})+\mathrm{H}_{2} \mathrm{O}$

Entretanto, o LiOH na solução encontra-se em excesso, para evitar a volatilização de $\mathrm{Br}_{2}$ em meio ácido ${ }^{11}$. No procedimento analítico na qual é empregada, essa solução é responsável pela conversão de espécies nitrogenadas a $\mathrm{N}_{2}$ segundo a reação (2)

$$
\begin{aligned}
\left(\mathrm{NH}_{4}\right)_{2} \mathrm{SO}_{4}(\mathrm{aq})+3 \mathrm{LiBrO}(\mathrm{aq}) \rightarrow & \mathrm{N}_{2}(\mathrm{~g})+3 \mathrm{LiBr}(\mathrm{aq}) \\
& +\mathrm{H}_{2} \mathrm{SO}_{4}(\mathrm{aq})+3 \mathrm{H}_{2} \mathrm{O}
\end{aligned}
$$

Após o procedimento analítico, a solução empregada (2 mL) é retirada do tubo em $\mathrm{U}$ da linha de vácuo, através de lavagem com água desionizada, resultando em solução residual diluída em aproximadamente 5 vezes.

Visando recuperar o bromo contido nessa solução residual (concentração aproximada de $20 \mathrm{~g} \mathrm{~L}^{-1}$ de $\mathrm{Br}_{2}$ ), construiu-se o sistema apresentado na Figura 1. Nesse sistema, a solução residual alcalina é acidificada de acordo com a Equação (3)

$$
\begin{array}{r}
\mathrm{LiBr}(\mathrm{aq})+\mathrm{LiBrO}(\mathrm{aq})+2 \mathrm{LiOH}(\mathrm{aq})+2 \mathrm{H}_{2} \mathrm{SO}_{4}(\mathrm{aq}) \rightarrow \\
2 \mathrm{Li}_{2} \mathrm{SO}_{4}(\mathrm{aq})+\mathrm{Br}_{2}(\mathrm{~g})+3 \mathrm{H}_{2} \mathrm{O}
\end{array}
$$

$\mathrm{O} \mathrm{Br}_{2}$ liberado é arrastado através de fluxo de $\mathrm{N}_{2}$ até a solução de $\mathrm{LiOH} 10 \% \mathrm{~m} / \mathrm{v}$, a fim de promover reação estequiométrica (referente à Equação 1). Para facilitar a absorção do bromo pela solução de $\mathrm{LiOH}$, optou-se por realizar resfriamento ("trap" com gelo e água) nessa solução durante o procedimento de recuperação.

Para estabelecer as condições operacionais da linha de recupera- 


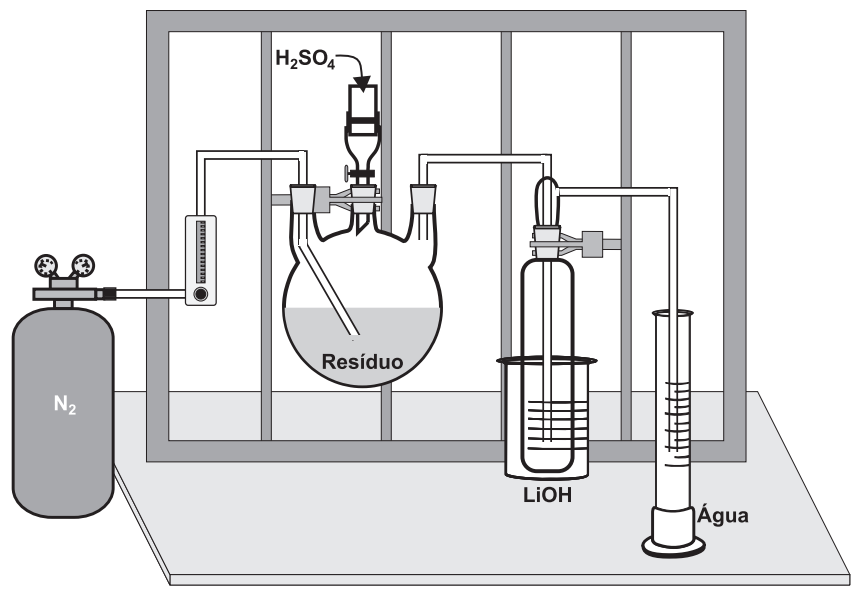

Figura 1. Linha especial em vidro construída para recuperação de bromo na forma de brometo e hipobromito de lítio

ção de bromo, foram realizados ensaios para avaliar a influência do fluxo de gás de arraste $\left(0,5,1\right.$ e $\left.2 \mathrm{~L} \mathrm{~min}^{-1}\right)$ e o tempo de reação na eficiência da recuperação do bromo.

As concentrações de $\mathrm{Br}_{2}$ das soluções residuais e recuperadas foram quantificadas seguindo a metodologia preconizada por $\mathrm{Creitz}^{12}$, empregando espectrofotômetro Perkin-Elmer Coleman 295.

Foram também realizadas determinações isotópicas de ${ }^{15} \mathrm{~N}$, no espectrômetro de massas ATLAS MAT modelo $\mathrm{CH} 4$, empregando o produto recuperado em comparação à solução preparada (reagente p.a) utilizada em rotina.

O resíduo ácido resultante do procedimento de recuperação de bromo foi neutralizado com solução de $\mathrm{NaOH} 10 \mathrm{~mol} \mathrm{~L}^{-1}$ e diluído antes de ser descartado na rede de coleta de esgoto.

Por fim, considerando-se que anualmente é realizada na instituição uma média de 5000 determinações isotópicas de ${ }^{15} \mathrm{~N}$, gerando um volume de $50 \mathrm{~L}$ de solução residual, foi possível estabelecer um balanço de custos atestando a viabilidade do sistema dimensionado.

\section{RESULTADOS E DISCUSSÃO}

A primeira etapa do trabalho visou adequar a metodologia proposta por Creitz ${ }^{12}$ para quantificação de bromo. Devido à característica deste elemento em formar as espécies brometo e hipobromito em meio alcalino, e para facilitar a apresentação e discussão dos resultados obtidos, optou-se em representar a sua concentração em $g$ $\mathrm{L}^{-1}$ de $\mathrm{Br}_{2}$ presentes na solução em análise. Ainda sobre a adequação metodológica, esclarece-se que a metodologia é baseada na medida da absorbância de suspensões de $\mathrm{AgBr}$ em comprimento de onda de $500 \mathrm{~nm}$.

Os primeiros ensaios atestaram que a absorbância variava significativamente em função do tempo, razão pela qual foram testadas as melhores situações nos intervalos de 5, 30, 60, 90 e 120 min após a formação da suspensão (reação com $\mathrm{AgNO}_{3}$ ). A observação dos resultados obtidos permitiu estabelecer que um intervalo de $60 \mathrm{~min}$ deve ser respeitado antes da realização das leituras de interesse, sendo as curvas de calibração construídas a partir de diluições da solução inicial ( $100 \mathrm{~g} \mathrm{~L}^{-1}$ de $\mathrm{Br}_{2}$ ), a qual foi utilizada como solução estoque.

Conhecendo-se a concentração de bromo nas soluções residuais armazenadas, realizaram-se os testes de recuperação empregando 1 L do resíduo, $40 \mathrm{~mL}$ de ácido sulfúrico comercial e um volume aproximado de $200 \mathrm{~mL}$ de solução de LiOH $10 \%$ m/v (variável em função da concentração de $\mathrm{Br}_{2}$ determinada na solução residual).
Nestas condições, variando entre 0,5 e $2 \mathrm{~L} \mathrm{~min}^{-1}$ o fluxo de $\mathrm{N}_{2}$, observou-se que o tempo de reação varia em função do fluxo de gás de arraste, não influenciando, entretanto, na reação entre o $\mathrm{LiOH}$ e o $\mathrm{Br}_{2}$ arrastado. Como seria inviável aumentar ainda mais o fluxo de $\mathrm{N}_{2}$, pois acarretaria em formação de pressão elevada no sistema, definiu-se pelo emprego de fluxo da ordem de $2 \mathrm{~L} \mathrm{~min}^{-1}$.

Estabelecidas as condições anteriormente especificadas (fluxo: $2 \mathrm{~L} \mathrm{~min}^{-1}$ de $\mathrm{N}_{2}$; tempo de reação: $2,5 \mathrm{~h}$ ), foram realizados 5 testes a fim de se verificar a eficiência da recuperação de bromo. Os resultados experimentais mostraram que o sistema apresenta eficiência média de $82 \pm 2 \%$. Desta forma, como a solução recuperada apresentou concentração de bromo $82 \%$ da esperada, e sendo isto indesejável, a alternativa encontrada foi reduzir o volume de solução de LiOH $10 \% \mathrm{~m} / \mathrm{v}$ nessa mesma proporção, ou seja, empregar $82 \%$ do volume estequiométrico. Ensaios realizados sob essas condições apresentaram resultados satisfatórios, como pode ser notado na Figura 2, que ilustra a construção de duas curvas de calibração elaboradas empregando as soluções recuperada e preparada (reagente p.a.).

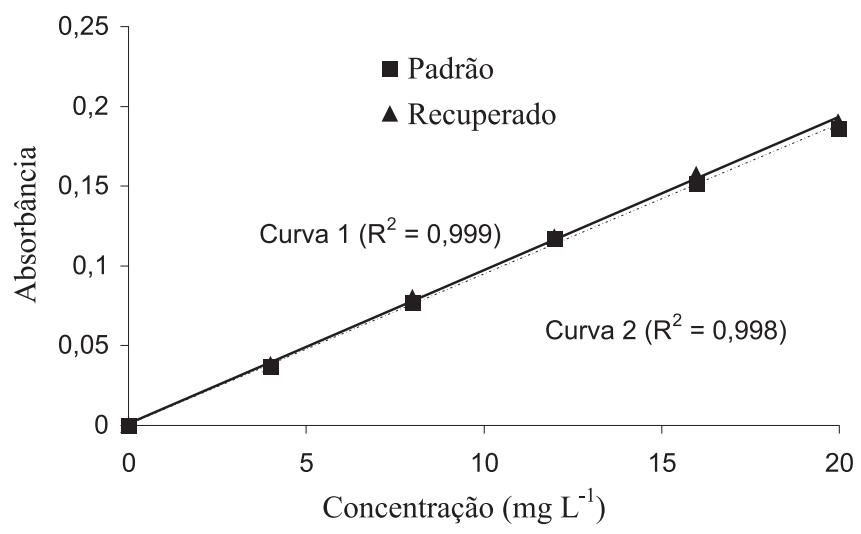

Figura 2. Curvas de calibração elaboradas empregando soluções recuperada (Curva 1) e preparada (Curva 2)

Para aferir a qualidade do produto recuperado, procederam-se determinações isotópicas de ${ }^{15} \mathrm{~N}$ em $\left({ }^{15} \mathrm{NH}_{4}\right)_{2} \mathrm{SO}_{4}$ enriquecido em 0,5 e 2 átomos \% empregando as soluções recupera e preparada (reagente p.a.). Os resultados, listados na Tabela 1, não diferiram estatisticamente a nível de 95\% de probabilidade (teste T de Student), o que corrobora o emprego do procedimento de recuperação em rotina.

Tabela 1. Determinações isotópicas de ${ }^{15} \mathrm{~N}$ realizadas em $\left({ }^{15} \mathrm{NH}_{4}\right)_{2} \mathrm{SO}_{4}$ enriquecido em 0,5 e 2 átomos \%, empregando soluções recuperada e preparada (5 repetições)

\begin{tabular}{lcc}
\hline Solução & $\left({ }^{15} \mathrm{NH}_{4}\right)_{2} \mathrm{SO}_{4} 0.5$ at $\%$ & $\left({ }^{15} \mathrm{NH}_{4}\right)_{2} \mathrm{SO}_{4} 2$ at $\%$ \\
\hline Preparada & $0,500 \pm 0,002$ & $1,977 \pm 0,010$ \\
Recuperada & $0,502 \pm 0,002$ & $1,961 \pm 0,005$ \\
\hline
\end{tabular}

O cálculo do número de determinações (ND) de ${ }^{15} \mathrm{~N}$ possíveis de serem realizadas, partindo de $1 \mathrm{~L}$ de solução preparada com reagente p.a. (volume suficiente para 500 determinações), após sucessivas recuperações, é dado pela Equação (4)

$\mathrm{Y} *(\mathrm{Ef})^{\mathrm{n}}=2$

onde Y é o volume de solução inicial, Ef é a eficiência da recuperação e n o número de ciclos de recuperação, e, com isso, o ND é calculado segundo a Equação (5)

$\mathrm{ND}=1 / 2 *\left[\mathrm{Y}+(\mathrm{Y} * \mathrm{Ef})+(\mathrm{Y} * \mathrm{Ef})^{2}+(\mathrm{Y} * \mathrm{Ef})^{3} \ldots+(\mathrm{Y} * \mathrm{Ef})^{\mathrm{n}}\right]$ 
Empregando-se as equações supracitadas, observa-se que, partindo de apenas $1 \mathrm{~L}$ de solução preparada, volume necessário para realizar 500 determinações, e efetuando-se sucessivas recuperações nos resíduos analíticos, é possível realizar um total de aproximadamente 2700 determinações de ${ }^{15} \mathrm{~N}$. Com isso, elaborou-se uma planilha de custos para ilustrar melhor o considerável retorno financeiro advindo desta atividade. Na Tabela 2 são apresentados os custos de reagentes empregados no sistema de recuperação de bromo.

Tabela 2. Custo referente à recuperação de bromo (base de cálculo 1 ano ou 5000 determinações de ${ }^{15} \mathrm{~N}$ )

\begin{tabular}{lr}
\hline Materiais e equipamentos & $\mathrm{R} \$ *$ \\
\hline Gás de arraste & 180,00 \\
Ácido sulfúrico & 10,00 \\
Hidróxido de sódio & 5,00 \\
Amortização (linha de recuperação) & 250,00 \\
\hline Total & 445,00 \\
\hline
\end{tabular}

* valores estimados considerando $82 \%$ de eficiência de recuperação.

O custo da montagem da linha de recuperação foi de $\mathrm{R} \$ 2.500,00$, e considerou-se em 10 anos o tempo de amortização do sistema, representando custo anual de $\mathrm{R} \$ 250,00$. Não se considerou, para efeito de cálculo, hora/atividade de técnico capacitado para efetuar os procedimentos de recuperação. Isto se justifica em virtude desta atividade não ser muito morosa, demandando esforços similares àqueles dispensados quando do preparo empregando reagentes p.a., sendo que, esta última, deixará de ser realizada com freqüência.

Considerando-se o elevado custo do bromo líquido ( $\mathrm{R} \$ 185,00$ o frasco de $50 \mathrm{~g}$ ), reagente empregado de elevada pureza, tem-se que, para realizar as mesmas 5000 determinações, o custo apenas deste reagente é de $\mathrm{R} \$ 3.700,00$. Esse valor, quando comparado ao total demandado pelo procedimento de recuperação, resulta em mais de $\mathrm{R} \$ 3.200,00$ ano $^{-1}$ de economia para a instituição.

Ressalta-se, ainda sobre a Tabela 2, que o hidróxido de sódio computado é empregado na neutralização da solução ácida resultante do procedimento de recuperação de bromo. Após a neutralização, a solução é descartada numa caixa de contenção existente no LIECENA/USP. Neste laboratório, são produzidos compostos enriquecidos em ${ }^{15} \mathrm{Ne} \mathrm{e}^{34} \mathrm{~S}$, e também água desionizada, empregando a técnica de cromatografia de troca iônica, e a referida caixa de contenção é utilizada para neutralizar volumes significativos (200 L) de água de lavagem das resinas (soluções ácidas e alcalinas). Com isso, a solução residual ácida, que ainda contém bromo solúvel, é neutralizada e diluída antes de ser descartada na rede de coleta de esgotos. Esse cuidado é tomado mesmo considerando que a Resolução CONAMA 20, em seu Artigo n ${ }^{\circ} 21$, que estabelece os padrões de emissão para efluentes de qualquer fonte poluidora nos corpos de água, não recomenda limite para concentração de bromo em efluentes ${ }^{13}$. Essa mesma resolução explicita que não é permitida a diluição de solução residual para que se atenda aos parâmetros de emissão estabeleci- dos. Entretanto, deve-se esclarecer que, no caso da diluição da solução contendo bromo, não é consumida água limpa, mas sim outros volumes de água de lavagem residual.

No sistema desenvolvido, a substituição da armadilha química contendo solução de hidróxido de lítio $10 \%(\mathrm{~m} / \mathrm{v})$ por um sistema criogênico (reservatório especial de vidro com banho de $\mathrm{CO}_{2}(\mathrm{~s})$ e etanol à temperatura de $-73{ }^{\circ} \mathrm{C}$ ) deve permitir a recuperação do bromo no estado líquido.

\section{CONCLUSÃO}

Os resultados experimentais verificados mostram que o desenvolvimento da linha de recuperação foi uma importante contribuição para a implantação do PGRQ-CENA/USP. A utilização deste sistema em rotina tem permitido a minimização do volume de resíduo na instituição, acarretando ainda retorno financeiro para o LIE-CENA/ USP. Entretanto, além da questão financeira, deve-se considerar que, infelizmente, custo ambiental não é mensurável, e não aparece nunca em uma planilha de custos. Caso isso fosse possível, trabalhos desta natureza seriam ainda mais valorizados.

Deve-se ressaltar, também, que embora tenha sido desenvolvido para recuperar o bromo contido em uma solução residual específica, o mesmo sistema pode ser útil no tratamento de outros resíduos que contenham esse mesmo elemento. A recuperação de bromo na forma líquida também pode ser realizada com a adaptação proposta.

\section{AGRADECIMENTOS}

Os autores agradecem à Fundação de Amparo a Pesquisa do Estado de São Paulo (FAPESP) e à Coordenação de Aperfeiçoamento de Pessoal de Nível Superior (CAPES) pelo auxílio financeiro concedido, e ao desenhista J. G. Brancalion pela editoração das figuras.

\section{REFERÊNCIAS}

1. Jardim, W. F.; Quim. Nova 1998, 21, 671.

2. Ashbrook, P. C.; Reinhardt, P. A.; Environ. Sci. Technol. 1985, 19, 1150.

3. Bendassolli, J. A.; Maximo, E.; Tavares, G. A.; Ignoto, R. F.; Quim. Nova 2003, 26, 612

4. Cunha, C. J. da; Quim. Nova 2001, 24, 424.

5. Amaral, S. T.; Machado, P. F. L.; Peralba, M. do C. R.; Camara, M. R.; Santos, T. dos; Berleze, A. L.; Falcão, H. L.; Martinelli, M.; Gonçalves, R. S.; Oliveira, E. R. de; Brasil, J. L.; Araújo, M. A. de; Borges, A. C. A.; Quim. Nova 2001, 24, 419.

6. Rittenberg, D.; The preparation and mensurement of the isotopic tracers, J. M. Eduards: Michigan, 1946.

7. Bendassolli, J. A.; Mortati, J.; Trivelin, P. C. O.; Ignoto, R. F.; Bonassi, J. A.; Tavares, G. A.; Quim. Nova 2002, 25, 312.

8. Manahan, S. E.; Toxicological Chemistry, $2^{\text {th }}$ ed., Lewis: Michigan, 1992

9. Ward, R. W.; DeGrave, G. M.; Water Res. Bull. 1978, 14, 696.

10. Fisher, D. J.; Burton, D. T.; Yonkos, L. T.; Turley, S. D.; Ziegler, G. P.; Water Res. 1999, 33, 760.

11. Marczenko, Z.; Separation and spectrophotometric determination of elements, John Wiley \& Sons: New York, 1986.

12. Creitz, E. C.; Anal. Chem. 1965, 37, 1690.

13. http://www.mma.gov.br/port/conama, acessada em Janeiro 2003. 